\title{
Changes in Grain Yield and Root Morphology and Physiology of Mid-Season Rice in the Yangtze River Basin of China During the Last 60 Years
}

\author{
Lijun Liu ${ }^{1}$, Hao Zhang ${ }^{1}$, Chengxin Ju${ }^{1}$, Yiwei Xiong ${ }^{1}$, Jinglong Bian ${ }^{1}$, Buhong Zhao ${ }^{2} \&$ Jianchang Yang $^{1}$ \\ ${ }^{1}$ Key Laboratory of Crop Genetics and Physiology of Jiangsu Province/Key Laboratory of Crop Physiology, \\ Ecology and Cultivation in Middle and Lower Reaches of Yangtze River of Ministry of Agriculture, Yangzhou \\ University, Yangzhou, China \\ ${ }^{2}$ LiXiahe Agricultural Institute of Jiangsu Province, Yangzhou, Jiangsu, China \\ Correspondence: Jianchang Yang, Department of Agronomy, Agricultural Collelge, Yangzhou University, \\ Yangzhou 225009, China. Tel: 86-514-8797 9317. E-mail: jcyang@yzu.edu.cn
}

Received: March 7, 2014 Accepted: April 23, 2014 Online Published: June 15, 2014

doi:10.5539/jas.v6n7p1 URL: http://dx.doi.org/10.5539/jas.v6n7p1

\begin{abstract}
The genetic improvement (GI) plays an important role in yield increase in rice in the Yangtze River Basin of China, and rice roots might have a crucial function during the GI. However, little is known what changes in root physiology and morphology were during the GI in this area and how they were related with grain yield. In this study, 24 typical mid-season rice cultivars (including hybrid combinations) applied in the area during the last 60 years were grown in the field, and they were classified into six types according to their application decades in which the cultivars were predominantly used. Changes in morphological and physiological traits of roots and their relationships with grain yield during the GI were investigated. Results showed that grain yield was significantly increased. Increase in grain yield was attributed mainly to the increase in total number of spikelets, which resulted mainly from a large panicle. The root dry weight, root length, root diameter, root oxidation activity, total absorbing surface area and active absorbing surface area of roots were also increased with the GI. Regression analysis showed that the root dry weight, root length, root diameter, root oxidation activity, total absorbing surface area and active absorbing surface area of roots were very significantly and positively correlated with grain yield. The results suggest that the GI improves root morph-physiological traits which benefits shoot growth, leading to a higher grain yield for the modern cultivars, especially for super rice cultivars.
\end{abstract}

Keywords: mid-season rice, genetic improvement, root morphology/physiology, grain yield

\section{Introduction}

The genetic improvement (GI) in rice plays a crucial role in the yield increase in China and even in the world (Cheng et al., 2007; Zhang et al., 2007). As an integral part of plant organs, plant roots are involved in acquisition of water and nutrients, synthesis of plant hormones, organic acids and amino acids, and anchorage of plants (Fitter et al., 2002; Inukai et al., 2004). Rice roots might play an important role in the yield increase during the GI. However, compared with well-studied above-ground traits like photosynthesis (Lawson et al., 2011; Gu et al., $2012,2014)$, little is known about the changes in morphological and physiological traits of roots during the GI and their relationships with grain yield in rice.

The Yangtze River Basin of China is one of the most important rice planting areas in China. The rice cropping systems are diverse in this area, such as double rice cropping systems, single mid-season or late-season rice cropping systems. Indica and japonica rice cultivars co-exist in most area of this basin. Mid-season rice is the dominant one among all the rice planting systems (China National Rice Research Institute, 1988; Zheng et al., 2003). There have been many reports on root traits for high-yielding rice cultivars (Harada et al., 1994; Kang et al., 1994; Ling, 2000; Liu et al., 2003). Ling (2000) proposed that a high-yielding rice cultivar should have a great and vigorous root system. It was also reported that the large aboveground biomass of japonicalindica hybrids was mainly attributed to their large root system (Harada et al., 1994; Kang et al., 1994; Liu et al., 2003). Although the differences in root traits (Dong et al., 2002; Wu et al., 2001; Zhu et al., 2000), grain yield, grain quality and plant types between hybrid and inbred rice of mid-season indica cultivars have been studied (Yang et al., 2006; Zhang et 
al., 2009), there were few studies on the changes in morphological and physiological traits of roots of mid-season rice cultivars and their relationships with grain yield during the GI. In this study, 24 typical mid-season rice cultivars (including hybrid combinations) applied in the Yangtze River Basin of China during the last 60 years were grown in the paddy field, and changes in morphological and physiological traits of roots and their relationships with grain yield during GI were investigated. The objectives of this study were to: a) Investigate the changes in root morphology and physiology of mid-season rice during the last 60 years, and b) to evaluate the relationships of the morphological and physiological traits of roots with grain yield.

\section{Method}

\subsection{Plant Materials and Cultivation}

The experiment was conducted at a research farm belonging to the key laboratory of crop genetics and physiology of Yangzhou University, Jiangsu Province, China $\left(32^{\circ} 30^{\prime} \mathrm{N}, 119^{\circ} 25^{\prime} \mathrm{E}\right)$ during the rice growing season (May to October) in 2008, and repeated in 2009. The preceding crop was wheat (Triticum aestivum L.) in both years. The soil was a sandy loam [Typic fluvaquents, Etisols (U.S.taxonomy)] with $2.04 \mathrm{~g} \mathrm{~kg}^{-1}$ organic matter, $105.1 \mathrm{mg} \mathrm{kg}^{-1}$ alkali hydrolysable nitrogen (N), $30.7 \mathrm{mg} \mathrm{kg}^{-1}$ Olsen-phosphorus (P), and $87.2 \mathrm{mg} \mathrm{kg}^{-1}$ exchangeable potassium (K). 24 typical mid-season rice cultivars (12 indica rice and 12 japonica rice cultivars, including hybrid combinations) cultivated in the Yangtze River Basin of China during the last 60 years were selected, and classified into six types according to their application decades (Table 1). The high-yielding cultivars of 1950s were selected by the local farmers and rice breeders. The five super rice cultivars in this study were all examined and approved by the Ministry of Agriculture of China. The rest cultivars were chosen because they had large planting acreage (> $\left.6.67 \times 10^{4} \mathrm{ha}\right)$.

Table 1. The tested mid-season indica and japonica rice cultivars

\begin{tabular}{clll}
\hline Application decades & \multicolumn{1}{c}{ Years of application } & Indica rice cultivars & Japonica rice cultivars \\
\hline 1950s & $1950-1960$ & Yintiaoxian & Huangkezao \\
& $1950-1960$ & Nanjing 1 & Guihuaqiu \\
1960s & $1960-1970$ & Taizhongxian & Jinnanfeng \\
& $1960-1970$ & Nanjing 11 & Chunfeng \\
$1970 \mathrm{~s}$ & $1970-1980$ & Zhenzhu'ai & Xudao 2 \\
& $1970-1980$ & IR24 & Liming \\
$1980 \mathrm{~s}$ & $1980-1990$ & Yangdao 2 & Suxiejing \\
& $1980-1990$ & Yangdao 6 & Yanjing 1 \\
1990s & $1990-2000$ & Shanyou 63 (Hybrid) & Zhendao 88 \\
& $1990-2000$ & Liangyoupeijiu (Hybrid and Super) & Wuyujing 3 \\
2000s & $2000-$ & Yangliangyou 6 (Hybrid and Super) & Wujing 15 (Super rice) \\
& $2000-$ & Ilyou 084 (Hybrid and Super) & Huaidao 9 (Super rice) \\
\hline
\end{tabular}

Across the two study years, seedlings were raised in the seedbed with sowing date on 8 May and transplanted on $8-10$ June at a hill spacing of $0.20 \mathrm{~m} \times 0.20 \mathrm{~m}$ with two seedlings per hill. The experiments were laid out in a complete randomized block design with three replicates. Plot dimensions were $5 \mathrm{~m} \times 4 \mathrm{~m}$. The total $\mathrm{N}$ rate was 240 $\mathrm{kg} \mathrm{ha}^{-1} \mathrm{~N}$ and was applied as urea at pre-transplanting (1 day before transplanting), early tillering ( 7 days after transplanting) and panicle initiation stage (about 35 days after transplanting) with the proportion of $50 \%, 10 \%$ and $40 \%$, respectively. Phosphorus (40 kg ha ${ }^{-1}$ as single superphosphate) and potassium (40 kg ha ${ }^{-1}$ as $\left.\mathrm{KCl}\right)$ were applied as basal fertilizer at 1 day before transplanting. Except drainage at the mid-season, the field was continuously flooded with $2-3 \mathrm{~cm}$ water level until one week before harvest. Weeds, insects, and diseases were controlled as required to avoid yield loss.

\subsection{Sampling and Measurements}

At the stages of mid-tillering, panicle initiation, heading, grain filling ( $15 \mathrm{~d}$ after heading) and maturity, eight hills of plants were sampled for the measurements of aboveground biomass and root traits. For the measurement of aboveground biomass, all plant samples were separated into green leaf blades, stems (culms + sheaths), and 
panicles (heading and after heading). Dry matter of each component was determined by weighing after drying at $70{ }^{\circ} \mathrm{C}$ to constant weight. For each root sampling, a block of soil $(20 \mathrm{~cm} \times 20 \mathrm{~cm} \times 20 \mathrm{~cm})$ around each individual hill was dug up using a sampling core. The roots were carefully rinsed with a hydropneumatic device and detached from their nodal bases and then weighed for determining fresh weight. Half of each root sample was used for measurements of root length and root diameter. The rest roots were dried in an oven at $70{ }^{\circ} \mathrm{C}$ to constant weight and weighed. To measure root length and diameter, roots were arranged and floated on shallow water in a glass tray $(30 \mathrm{~cm} \times 30 \mathrm{~cm})$ and then scanned using a scanner (Epson Expression 1680 Scanner, Seiko Epson Corp., Tokyo, Japan) and analyzed using WinRHIZO Root Analyzer System (Regent Instruments Inc., Quebec, Canada). Specific root length was calculated from the root length and the root dry weigh (W), i.e. specific root length $\left(\mathrm{m} \mathrm{g}^{-1}\right)=$ root length $(\mathrm{m}) / \mathrm{W}(\mathrm{g})$. Root-shoot ratio was determined based on the dry weight of root and shoot. From panicle initiation, three hills of rice roots were sampled for the determination of root oxidation activity (ROA), total root absorbing surface area and active absorbing surface area. The ROA was determined by measuring oxidation of alphanaphthylamine ( $\alpha$-NA) according to the methods developed by Ota (1970) modified by Ramasamy et al. (1997), and was expressed as mg $\alpha$-NA per gram dry weight (DW) per hour (mg $\alpha$-NA g ${ }^{-1} \mathrm{DW} \mathrm{h}^{-1}$ ). The total root absorbing surface area and active absorbing surface area were determined referring to the methods of Xiao et al. (2005).

Grain yield was determined from a harvest area of $5 \mathrm{~m}^{2}$ in each plot at physiological maturity of each cultivar and adjusted to a moisture content of $0.14 \mathrm{~g} \mathrm{H}_{2} \mathrm{O} \mathrm{g}^{-1}$ fresh weight. Yield components, i.e., number of panicles per square meter, percentage of filled grains and 1000-grain weight, were determined from plants of $1 \mathrm{~m}^{2}$ that was representative and without obvious bad spots or damage in each plot. The percentage of filled grains (specific gravity $\geq 1.06 \mathrm{~g} \mathrm{~cm}^{-3}$ ) was determined as the ratio of filled grains to total number of spikelets. The number of spikelets per panicle was calculated from the grain yield, grain weight $\left(0.14 \mathrm{~g} \mathrm{H}_{2} \mathrm{O} \mathrm{g} \mathrm{g}^{-1}\right.$ moisture content), and percentage of filled grains, i.e., number of spikelets per panicle $=$ grain yield per square meter/(number of panicles per square meter $\times 1000$-grain weight $\times$ percentage of filled grains).

\subsection{Statistical Analysis}

Analysis of variance was performed using SAS/STAT statistical analysis package (version 6.12, SAS Institute, Cary, NC). Data were tested with a post-hoc least significant difference test at the $P=0.05$ level $\left(\mathrm{LSD}_{0.05}\right)$. A GLM analysis of variance procedure was run to identify significant source of variance. Sources of variation were partitioned between effects due to year, cultivars released at different decades, cultivars nested within decade, and residual error. All variables were subjected to normality and variance homogeneity tests prior to GLM analysis. Graphs were drawn using SigmaPlot 10.0 (SPSS Inc., Point Richmond, CA, USA). Correlation analysis was used to evaluate the relationship of morphological and physiological traits of roots with grain yield.

\section{Results}

\subsection{Grain Yield and Its Components}

The genetic improvement (GI) of rice cultivars significantly increased grain yield (Tables 2 and 3). The average grain yield in each decade increased 1.90, 0.85, 0.85, 1.45 and $0.50 \mathrm{t} \mathrm{ha}^{-1}$ from $1950 \mathrm{~s}$ to $2000 \mathrm{~s}$, and the increase percentages were $49.4 \%, 14.8 \%, 12.9 \%, 19.5 \%$ and $5.6 \%$, respectively. The increase in grain yield was mainly due to the increase in total number of spikelets (no. of panicles $\times$ spikelets per panicle), which resulted mainly from the large panicle, especially for those super hybrid rice combinations. The average percentage of filled grains of four super rice cultivars in 2000 s was only $77.0 \%$ in the two study years, significantly lower than that of cultivars applied in 1990s (Tables 2 and 3). Low percentage of filled grains might be a major limiting factor to achieve high yield potential for these modern super rice cultivars. 
Table 2. Changes in grain yield, yield components during the genetic improvement of mid-season indica and japonica rice cultivars in 2008

\begin{tabular}{|c|c|c|c|c|c|c|c|}
\hline $\begin{array}{l}\text { Application } \\
\text { decades }\end{array}$ & Cultivars & $\begin{array}{l}\text { No. of panicles } \\
\qquad\left(\times 10^{4} \mathrm{ha}^{-1}\right)\end{array}$ & $\begin{array}{l}\text { Spikelets } \\
\text { per panicle }\end{array}$ & $\begin{array}{c}\text { Percentage of } \\
\text { filled grains }(\%)\end{array}$ & $\begin{array}{l}\text { Total spikelets } \\
\text { number } \\
\left(\times 10^{6} \mathrm{ha}^{-1}\right)\end{array}$ & $\begin{array}{l}\text { 1000-grain } \\
\text { weight }(\mathrm{g})\end{array}$ & $\begin{array}{l}\text { Yield } \\
\left(\mathrm{t} \mathrm{ha}^{-1}\right)\end{array}$ \\
\hline \multirow[t]{5}{*}{$1950 \mathrm{~s}$} & Yintiaoxian & $286.2 \mathrm{~cd}$ & $103.3 \mathrm{ij}$ & $42.8 \mathrm{j}$ & $295.7 \mathrm{ijk}$ & $25.9 \mathrm{~cd}$ & $3.3 \mathrm{~m}$ \\
\hline & Nanjing 1 & $243.1 \mathrm{i}$ & 109.7 hi & $52.4 \mathrm{i}$ & $266.7 \mathrm{klm}$ & $25.0 \mathrm{f}$ & $3.5 \mathrm{~m}$ \\
\hline & Huangkezao & $243.1 \mathrm{i}$ & $109.7 \mathrm{hi}$ & $52.4 \mathrm{i}$ & $266.7 \mathrm{klm}$ & $25.0 \mathrm{f}$ & $3.5 \mathrm{~m}$ \\
\hline & Guihuaqiu & 216.81 & $109.3 \mathrm{hi}$ & $66.8 \mathrm{~h}$ & $236.9 \mathrm{~m}$ & $25.5 \mathrm{de}$ & $4.0 \mathrm{~lm}$ \\
\hline & Average & 247.3 & 108.0 & 53.6 & 266.5 & 25.4 & 3.6 \\
\hline \multirow[t]{5}{*}{$1960 \mathrm{~s}$} & Taizhongxian & $292.3 \mathrm{c}$ & $129.1 \mathrm{fg}$ & $75.4 \mathrm{efg}$ & $377.5 \mathrm{de}$ & $23.6 \mathrm{~h}$ & 6.7 ghi \\
\hline & Nanjing 11 & $304.6 \mathrm{~b}$ & $129.0 \mathrm{fg}$ & $76.2 \mathrm{ef}$ & $392.7 \mathrm{~d}$ & $26.2 \mathrm{bc}$ & 7.9 ef \\
\hline & Jinnanfeng & 284.0 cde & $112.0 \mathrm{hi}$ & $64.7 \mathrm{~h}$ & 318.2 ghi & $23.5 \mathrm{~h}$ & $4.8 \mathrm{kl}$ \\
\hline & Chunfeng & $232.8 \mathrm{j}$ & $118.5 \mathrm{gh}$ & $82.4 \mathrm{~cd}$ & $275.8 \mathrm{jkl}$ & $24.4 \mathrm{~g}$ & $5.6 \mathrm{jk}$ \\
\hline & Average & 278.4 & 122.2 & 74.7 & 341.1 & 24.4 & 6.2 \\
\hline \multirow[t]{5}{*}{$1970 \mathrm{~s}$} & Zhenzhu'ai & $233.9 \mathrm{j}$ & $131.3 \mathrm{efg}$ & $74.4 \mathrm{fg}$ & $306.7 \mathrm{hij}$ & $26.1 \mathrm{bc}$ & $6.0 \mathrm{ij}$ \\
\hline & IR24 & $310.8 \mathrm{ab}$ & $129.4 \mathrm{fg}$ & $75.1 \mathrm{fg}$ & $402.1 \mathrm{~d}$ & $24.5 \mathrm{~g}$ & $7.4 \mathrm{fg}$ \\
\hline & Xudao 2 & $269.8 \mathrm{fg}$ & $128.6 \mathrm{fg}$ & $82.1 \mathrm{~cd}$ & $346.7 \mathrm{efg}$ & $24.4 \mathrm{~g}$ & $7.0 \mathrm{gh}$ \\
\hline & Liming & $276.3 \mathrm{ef}$ & $94.1 \mathrm{j}$ & $84.5 \mathrm{abc}$ & $259.9 \mathrm{~lm}$ & $24.7 \mathrm{fg}$ & $5.4 \mathrm{jk}$ \\
\hline & Average & 272.7 & 120.8 & 79.0 & 328.8 & 25.0 & 6.5 \\
\hline \multirow[t]{5}{*}{$1980 \mathrm{~s}$} & Yangdao 2 & $258.5 \mathrm{~h}$ & $136.5 \mathrm{def}$ & $88.5 \mathrm{a}$ & 352.9 ef & $25.6 \mathrm{~d}$ & 7.9 ef \\
\hline & Yangdao 6 & $196.9 \mathrm{~m}$ & $169.9 \mathrm{c}$ & $71.7 \mathrm{~g}$ & $334.6 \mathrm{fgh}$ & $26.4 \mathrm{~b}$ & $7.8 \mathrm{ef}$ \\
\hline & Suxiejing & $311.6 \mathrm{ab}$ & $120.2 \mathrm{gh}$ & $87.7 \mathrm{ab}$ & $374.5 \mathrm{de}$ & $23.4 \mathrm{~h}$ & $6.3 \mathrm{hij}$ \\
\hline & Yanjing 1 & $319.3 \mathrm{a}$ & $126.3 \mathrm{fg}$ & 77.3 ef & $403.1 \mathrm{~d}$ & $25.6 \mathrm{~d}$ & 8.0 ef \\
\hline & Average & 271.6 & 138.2 & 81.3 & 366.3 & 25.2 & 7.5 \\
\hline \multirow[t]{5}{*}{$1990 \mathrm{~s}$} & Shanyou 63 & $258.5 \mathrm{~h}$ & $145.9 \mathrm{~d}$ & $87.5 \mathrm{ab}$ & $377.0 \mathrm{de}$ & $27.6 \mathrm{a}$ & $9.1 \mathrm{abcd}$ \\
\hline & Liangyoupeijiu & $221.5 \mathrm{lk}$ & $216.8 \mathrm{a}$ & $79.6 \mathrm{de}$ & $480.3 \mathrm{~b}$ & $26.1 \mathrm{bc}$ & $10.0 \mathrm{a}$ \\
\hline & Zhendao 88 & $279.6 \mathrm{de}$ & $142.1 \mathrm{de}$ & $84.0 \mathrm{bc}$ & $397.3 \mathrm{~d}$ & $25.1 \mathrm{ef}$ & $8.4 \mathrm{de}$ \\
\hline & Wuyujing 3 & $257.8 \mathrm{~h}$ & $169.6 \mathrm{c}$ & 77.5 ef & $437.2 \mathrm{c}$ & $25.6 \mathrm{~d}$ & 8.7 cde \\
\hline & Average & 254.3 & 168.6 & 82.1 & 423.0 & 26.1 & 9.0 \\
\hline \multirow[t]{5}{*}{$2000 \mathrm{~s}$} & $\begin{array}{c}\text { Yangliangyou } \\
6\end{array}$ & $244.1 \mathrm{i}$ & $164.3 \mathrm{c}$ & $85.5 \mathrm{abc}$ & $401.1 \mathrm{~d}$ & $26.1 \mathrm{bc}$ & $9.0 \mathrm{bcd}$ \\
\hline & II you 084 & $227.7 \mathrm{jk}$ & $211.1 \mathrm{a}$ & $78.6 \mathrm{def}$ & $480.9 \mathrm{~b}$ & $25.8 \mathrm{~cd}$ & $9.8 \mathrm{ab}$ \\
\hline & Wujing 15 & $282.8 \mathrm{de}$ & $183.3 \mathrm{~b}$ & $75.0 \mathrm{fg}$ & $518.2 \mathrm{a}$ & $24.3 \mathrm{~g}$ & $9.5 \mathrm{abc}$ \\
\hline & Huaidao 9 & $265.3 \mathrm{gh}$ & $193.2 \mathrm{~b}$ & $74.7 \mathrm{fg}$ & $512.5 \mathrm{a}$ & $25.1 \mathrm{ef}$ & $9.6 \mathrm{ab}$ \\
\hline & Average & 255.0 & 188.0 & 78.4 & 478.2 & 25.4 & 9.5 \\
\hline
\end{tabular}

Different letters indicate statistical significance at the $P=0.05$ level within the same column. 
Table 3. Changes in grain yield, yield components during the genetic improvement of mid-season indica and japonica rice cultivars in 2009

\begin{tabular}{|c|c|c|c|c|c|c|c|}
\hline $\begin{array}{l}\text { Application } \\
\text { decades }\end{array}$ & Cultivars & $\begin{array}{l}\text { No. of panicles } \\
\left(\times 10^{4} \mathrm{ha}^{-1}\right)\end{array}$ & $\begin{array}{l}\text { Spikelets } \\
\text { per panicle }\end{array}$ & $\begin{array}{l}\text { Percentage of } \\
\text { filled grains } \\
(\%)\end{array}$ & $\begin{array}{l}\text { Total spikelets } \\
\text { number } \\
\left(\times 10^{6} \mathrm{ha}^{-1}\right)\end{array}$ & $\begin{array}{l}\text { 1000-grain } \\
\text { weight }(\mathrm{g})\end{array}$ & $\begin{array}{l}\text { Yield } \\
\left(\mathrm{t} \mathrm{ha}^{-1}\right)\end{array}$ \\
\hline \multirow[t]{5}{*}{$1950 \mathrm{~s}$} & Yintiaoxian & $263.8 \mathrm{f}$ & $98.0 \mathrm{q}$ & $55.7 \mathrm{kl}$ & $258.6 \mathrm{~m}$ & $25.6 \mathrm{def}$ & $3.7 \mathrm{p}$ \\
\hline & Nanjing 1 & $238.5 \mathrm{k}$ & $113.4 \mathrm{mno}$ & $58.9 \mathrm{jk}$ & $270.3 \mathrm{~lm}$ & $24.4 \mathrm{i}$ & $3.9 \mathrm{p}$ \\
\hline & Huangkezao & $276.9 \mathrm{e}$ & $106.9 \mathrm{p}$ & 54.11 & $296.1 \mathrm{k}$ & $24.4 \mathrm{i}$ & $3.9 \mathrm{p}$ \\
\hline & Guihuaqiu & 249.2 hi & 109.6 op & $72.8 \mathrm{gh}$ & 273.21 & $25.6 \mathrm{def}$ & $5.1 \mathrm{n}$ \\
\hline & Average & 257.1 & 107.0 & 60.4 & 274.6 & 25.0 & 4.1 \\
\hline \multirow[t]{5}{*}{$1960 \mathrm{~s}$} & Taizhongxian & $243.9 \mathrm{j}$ & $122.8 \mathrm{k}$ & $74.3 \mathrm{fgh}$ & $299.4 \mathrm{k}$ & 24.8 ghi & $5.5 \mathrm{~lm}$ \\
\hline & Nanjing 11 & $217.7 \mathrm{n}$ & $135.6 \mathrm{i}$ & $68.8 \mathrm{i}$ & $295.2 \mathrm{k}$ & $26.2 \mathrm{bcd}$ & $5.3 \mathrm{mn}$ \\
\hline & Jinnanfeng & $295.4 \mathrm{c}$ & $107.9 \mathrm{p}$ & $60.5 \mathrm{j}$ & $318.6 \mathrm{ij}$ & $23.6 \mathrm{j}$ & $4.6 \mathrm{o}$ \\
\hline & Chunfeng & $264.6 \mathrm{f}$ & 111.8 nop & $79.0 \mathrm{cde}$ & $295.8 \mathrm{k}$ & $24.4 \mathrm{i}$ & $5.7 \mathrm{kl}$ \\
\hline & Average & 255.4 & 119.5 & 70.6 & 302.3 & 24.8 & 5.3 \\
\hline \multirow[t]{5}{*}{$1970 \mathrm{~s}$} & Zhenzhu'ai & $246.3 \mathrm{ij}$ & $127.8 \mathrm{j}$ & $81.0 \mathrm{bcd}$ & $314.8 \mathrm{j}$ & 25.4 efg & $6.5 \mathrm{j}$ \\
\hline & IR24 & $256.3 \mathrm{~g}$ & $144.3 \mathrm{~h}$ & $85.0 \mathrm{a}$ & $369.7 \mathrm{~h}$ & $24.4 \mathrm{i}$ & $7.7 \mathrm{~g}$ \\
\hline & Xudao 2 & $280.0 \mathrm{de}$ & $118.1 \mathrm{klm}$ & $83.7 \mathrm{ab}$ & $330.5 \mathrm{i}$ & $24.5 \mathrm{hi}$ & $6.8 \mathrm{ij}$ \\
\hline & Liming & $249.2 \mathrm{hi}$ & $115.4 \mathrm{lmn}$ & $82.8 \mathrm{abc}$ & $287.6 \mathrm{k}$ & 24.8 ghi & $5.9 \mathrm{k}$ \\
\hline & Average & 257.9 & 126.4 & 83.1 & 325.7 & 24.8 & 6.7 \\
\hline \multirow[t]{5}{*}{$1980 \mathrm{~s}$} & Yangdao 2 & $237.7 \mathrm{kl}$ & $163.2 \mathrm{e}$ & $73.5 \mathrm{gh}$ & $388.0 \mathrm{f}$ & $25.3 \mathrm{efg}$ & $7.2 \mathrm{~h}$ \\
\hline & Yangdao 6 & 248.7 hi & $150.4 \mathrm{~g}$ & $81.4 \mathrm{abcd}$ & $374.1 \mathrm{gh}$ & $26.6 \mathrm{ab}$ & $8.7 \mathrm{~d}$ \\
\hline & Suxiejing & $323.1 \mathrm{a}$ & $120.3 \mathrm{kl}$ & $72.0 \mathrm{hi}$ & $388.6 \mathrm{f}$ & 24.9 ghi & 8.1 ef \\
\hline & Yanjing 1 & $307.7 \mathrm{~b}$ & $117.7 \mathrm{klm}$ & $78.1 \mathrm{def}$ & $362.3 \mathrm{~h}$ & $25.8 \mathrm{cde}$ & $7.0 \mathrm{hi}$ \\
\hline & Average & 279.3 & 137.9 & 76.3 & 378.2 & 25.7 & 7.4 \\
\hline \multirow[t]{5}{*}{$1990 \mathrm{~s}$} & Shanyou 63 & $226.6 \mathrm{~m}$ & $170.0 \mathrm{~d}$ & $84.8 \mathrm{ab}$ & $385.2 \mathrm{fg}$ & $26.7 \mathrm{ab}$ & $7.3 \mathrm{~h}$ \\
\hline & Liangyoupeijiu & 211.60 & $225.4 \mathrm{~b}$ & $74.3 \mathrm{fgh}$ & $477.0 \mathrm{c}$ & $26.4 \mathrm{abc}$ & $9.3 \mathrm{bc}$ \\
\hline & Zhendao 88 & $283.5 \mathrm{~d}$ & $155.9 \mathrm{f}$ & $82.6 \mathrm{abc}$ & $441.8 \mathrm{~d}$ & $25.3 \mathrm{efg}$ & $9.2 \mathrm{c}$ \\
\hline & Wuyujing 3 & $251.4 \mathrm{~h}$ & $161.1 \mathrm{e}$ & 76.3 efg & $405.0 \mathrm{e}$ & $25.6 \mathrm{def}$ & $7.9 \mathrm{fg}$ \\
\hline & Average & 243.2 & 178.1 & 79.5 & 427.2 & 26.0 & 8.8 \\
\hline \multirow[t]{5}{*}{$2000 \mathrm{~s}$} & Yangliangyou 6 & 233.81 & $165.8 \mathrm{de}$ & $79.3 \mathrm{cde}$ & $387.6 \mathrm{f}$ & $26.9 \mathrm{a}$ & $8.3 \mathrm{e}$ \\
\hline & II you 084 & $226.2 \mathrm{~m}$ & $213.9 \mathrm{c}$ & $76.6 \mathrm{efg}$ & $483.7 \mathrm{c}$ & $26.6 \mathrm{ab}$ & 9.9 a \\
\hline & Wujing 15 & $218.5 \mathrm{n}$ & $251.1 \mathrm{a}$ & $71.3 \mathrm{hi}$ & $548.5 \mathrm{a}$ & $24.5 \mathrm{hi}$ & $9.6 \mathrm{ab}$ \\
\hline & Huaidao 9 & $224.6 \mathrm{~m}$ & $227.0 \mathrm{~b}$ & $75.1 \mathrm{fgh}$ & $509.8 \mathrm{~b}$ & $25.1 \mathrm{fgh}$ & $9.6 \mathrm{ab}$ \\
\hline & Average & 225.8 & 214.4 & 75.6 & 482.4 & 25.8 & 9.3 \\
\hline
\end{tabular}

Different letters indicate statistical significance at the $P=0.05$ level within the same column.

Table 4 shows the computed $F$ values for the differences in grain yield and yield components of rice between/among application decades and rice cultivars within the application decades. The analysis indicated that there were significant differences $(P<0.05)$ in grain yield and its components except panicle number per unit area among decades. Variations due to experimental years $(2008,2009)$ and in interactions between experimental years and application decades were not significant (Table 4). 
Table 4. Analysis of variance of $F$-values of rice yield and components in mid-season indica and japonica rice cultivars between/among years and application decades

\begin{tabular}{lccccccc}
\hline $\begin{array}{l}\text { Source of } \\
\text { variation }\end{array}$ & $d f$ & $\begin{array}{c}\text { No. of } \\
\text { panicles }\end{array}$ & $\begin{array}{c}\text { Spikelets } \\
\text { per panicle }\end{array}$ & $\begin{array}{c}\text { Percentage of } \\
\text { filled grains }\end{array}$ & $\begin{array}{c}\text { Total } \\
\text { spikelets number }\end{array}$ & $\begin{array}{c}\text { 1000-grain } \\
\text { weight }\end{array}$ & Yield \\
\hline $\begin{array}{l}\text { Year } \\
(Y, 2008 \& 2009)\end{array}$ & 1 & NS & NS & NS & NS & NS & NS \\
$\begin{array}{l}\text { Application decade (D) } \\
\text { Cultivar }\end{array}$ & 5 & NS & $67.5^{* *}$ & $36.4^{* *}$ & $71.4^{* *}$ & $15.9^{* *}$ & $83.5^{* *}$ \\
$\begin{array}{l}\text { (within each decade) } \\
\text { Y } \times \mathrm{D}\end{array}$ & 23 & $2.37^{* *}$ & $5.15^{* *}$ & $3.37^{* *}$ & $4.37^{* *}$ & $11.09^{* *}$ & NS \\
\hline
\end{tabular}

NS, not significant at the $P=0.05$ level.

${ }^{* *}$ Significant at the $P=0.01$ level.

\subsection{Morphological Traits of Rice Roots and Shoot Dry Weight}

Similar results were obtained in the measurements of morphological traits (root dry weight, root length, root-shoot ratio and root diameter) and physiological traits (root oxidation activity and total absorbing surface area) of roots (Table 5). Since year was not a significant factor in the experiment, data of these root traits from the two years were averaged.

Table 5. Analysis of variance of $F$ values of main root traits in mid-season indica and japonica rice cultivars between/among years and application decades

\begin{tabular}{lccccccc}
\hline $\begin{array}{l}\text { Source of } \\
\text { variation }\end{array}$ & $d f$ & Root dry weight & $\begin{array}{c}\text { Root } \\
\text { length }\end{array}$ & $\begin{array}{c}\text { Root-shoot } \\
\text { ratio }\end{array}$ & $\begin{array}{c}\text { Diameter } \\
\text { of root }\end{array}$ & $\begin{array}{c}\text { Root } \\
\text { oxidation } \\
\text { activity }\end{array}$ & $\begin{array}{c}\text { Total } \\
\text { absorbing } \\
\text { surface } \\
\text { area }\end{array}$ \\
\hline $\begin{array}{l}\text { Year } \\
(Y, 2008 \& 2009)\end{array}$ & 1 & NS & NS & NS & NS & NS & NS \\
$\begin{array}{l}\text { Application decade (D) } \\
\begin{array}{l}\text { Cultivar } \\
\text { (within each decade) }\end{array}\end{array}$ & 5 & $57.2^{* *}$ & $19.7^{* *}$ & $75.5^{* *}$ & $36.9^{* *}$ & $67.5^{* *}$ & $37.2^{* *}$ \\
$\mathrm{Y} \times \mathrm{D}$ & 23 & $14.13^{* *}$ & $3.11^{* *}$ & $5.74^{* *}$ & $1.07^{*}$ & $5.92^{* *}$ & $7.83^{* *}$ \\
\hline
\end{tabular}

NS, not significant at the $P=0.05$ level.

** Significant at the $P=0.01$ probability level.

The root dry weight and root length at main growth stages were increased or significantly increased with the GI (Figure 1-A and B). Generally, there were no significant differences in shoot dry weight among cultivars used in different decades at the stages of mid-tillering and panicle initiation. From heading to maturity, the shoot dry weight was increased with the GI (Figure 2-A). The differences in root-shoot ratio between new released super rice cultivars (used in 2000s) and the preceding cultivars (from 1950s to 1990s) were much larger before the heading stage than at grain filling and mature stages (Figure 2-B). The root diameter at main growth stages was significantly increased (Figure 3-A), whereas the specific root length was significantly decreased (Figure 3-B) with the GI. 

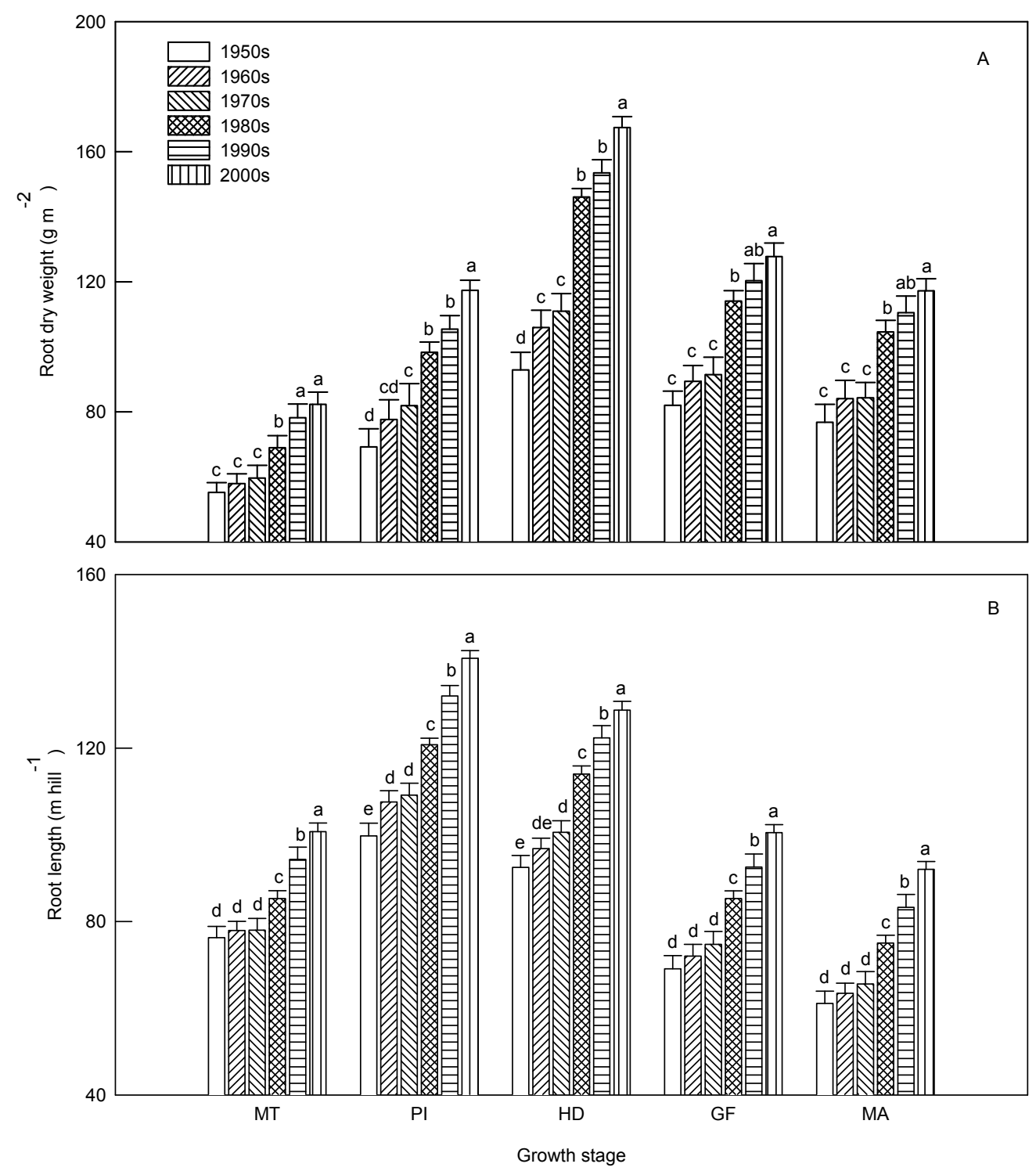

Figure 1. Seasonal changes in root dry weight (A) and root length (B) during the genetic improvement of mid-season indica and japonica rice cultivars. Data are averages observed for the two study years, because they showed the same tendencies. Vertical bars represent \pm SE of the mean. The SE was calculated across three replicates for each year and averaged for the 2 year. Different letters indicate statistical significance at the $P=0.05$ level within the same growth stage. MT: Middle tillering; PI: Panicle initiation; HD: Heading stage; GF: Grain filling stage; MA: Maturity. The same as below 

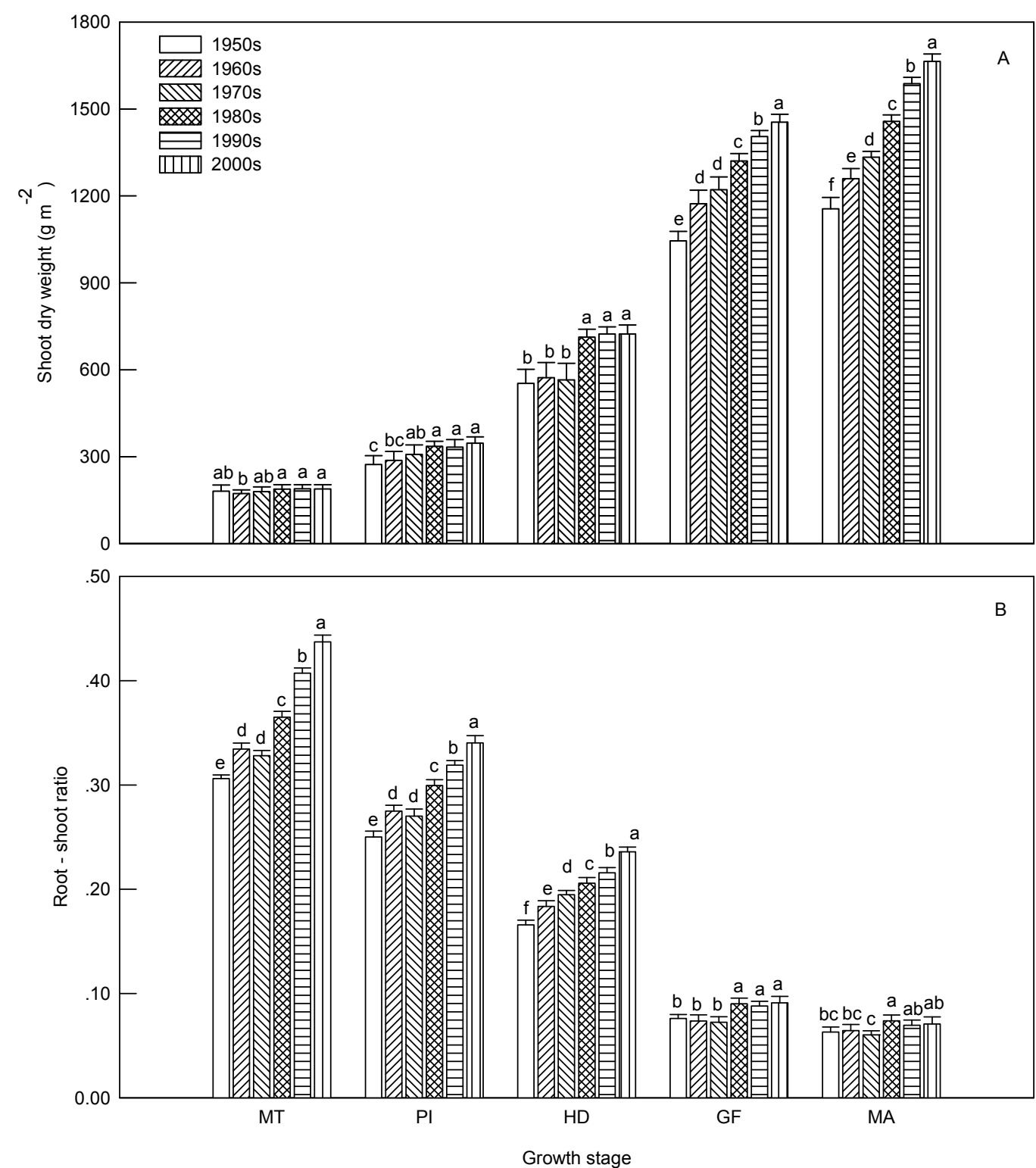

Figure 2. Seasonal changes in shoot dry weight (A) and root-shoot ratio (B) during the genetic improvement of mid-season indica and japonica rice cultivars 

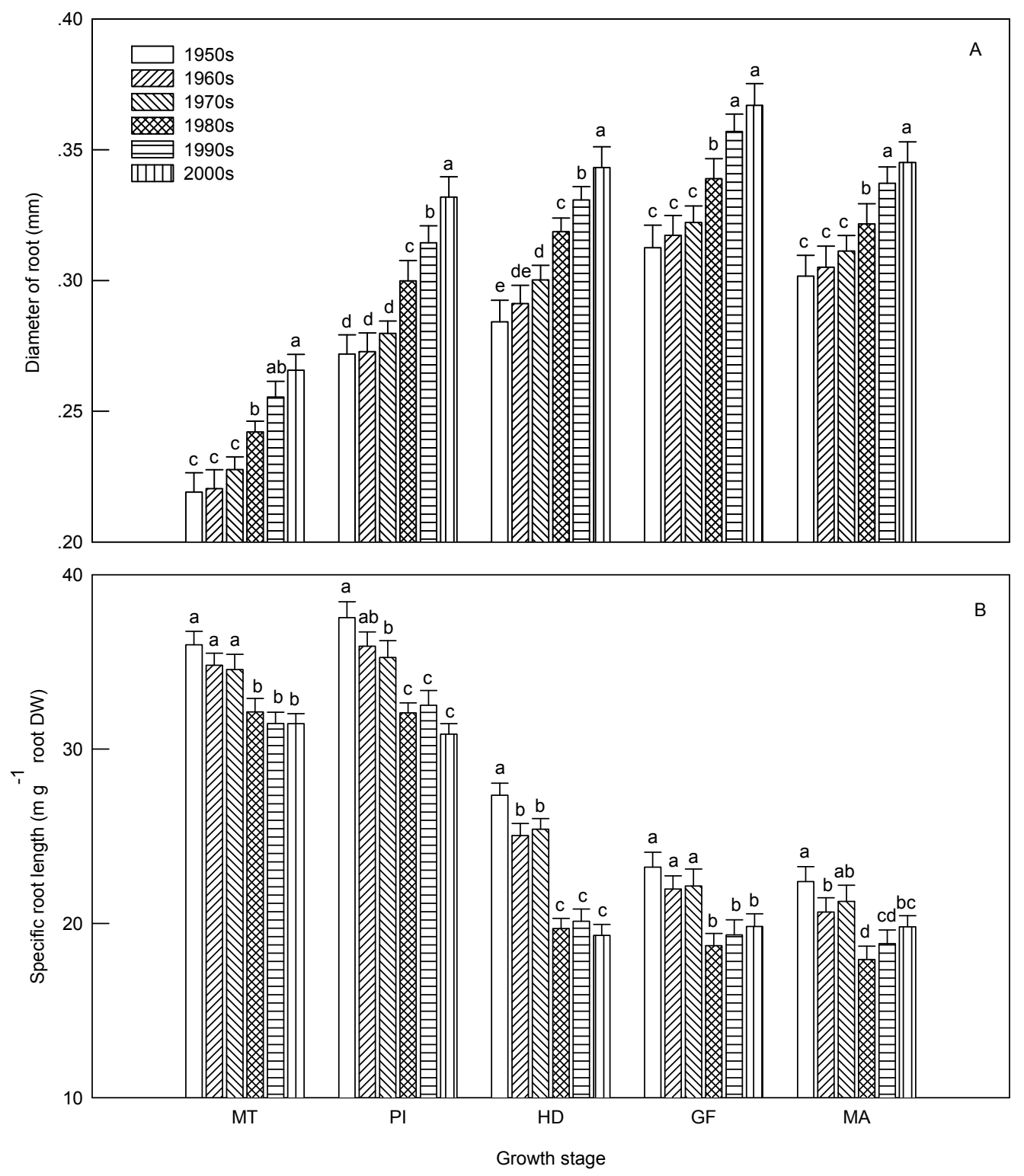

Figure 3. Seasonal changes in diameter of root (A) and specific root length (B) during the genetic improvement of mid-season indica and japonica rice cultivars

\subsection{Physiological Traits of Rice Roots}

Root oxidation activity (ROA) was significantly increased with the GI at the stages of panicle initiation and heading, and was significantly lower in super rice cultivars in 2000s than those cultivars in 1990s during the grain filling period (Figure 4-A), which might be an important reason for the lower percentage of filled grains in super rice cultivars.

The total absorbing surface area and active absorbing surface area of roots were increased or significantly increased with the GI before grain filling, and they declined faster in super rice cultivars after heading, when compared with any other cultivars (Figure 4-B and C). 

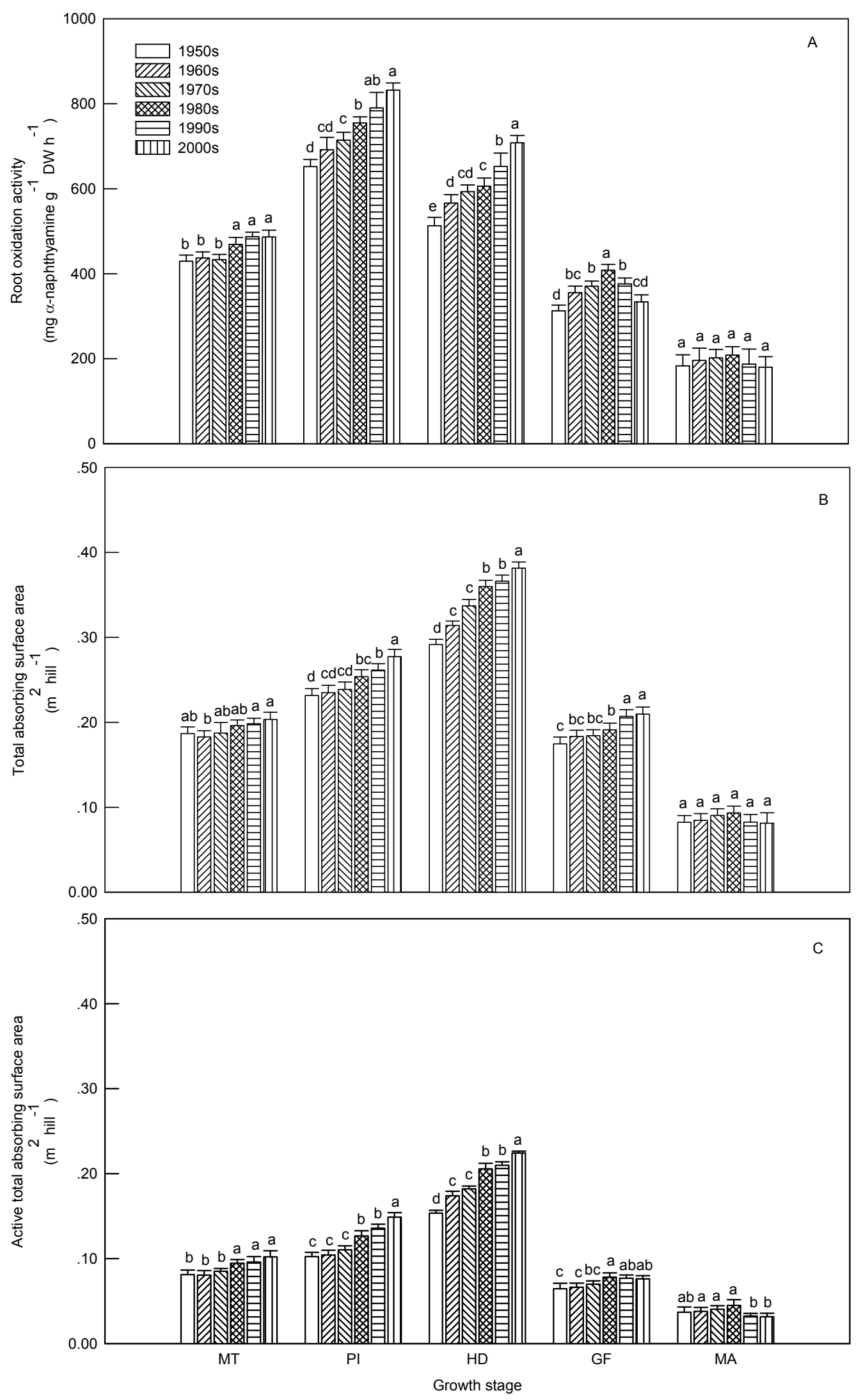

Figure 4. Seasonal changes in root oxidation activity (A), total absorbing surface area (B) and active absorbing surface area $(\mathrm{C})$ during the genetic improvement of mid-season indica and japonica rice cultivars 
Table 6. Relationships of root morphology and physiology parameters with yield and its components during the genetic improvement of mid-season indica and japonica rice cultivars. Data are averages observed for the two study years because they showed the same tendencies. MT: Middle tillering; PI: Panicle initiation; HD: Heading stage; GF: Grain filling stage; MA: Maturity. Data are from Figures 1-4 and Tables 2-3. The coefficient $r$ is calculated. ** and * represents significant respectively at the $P=0.01$ and $P=0.05$ level $(\mathrm{n}=24)$

\begin{tabular}{|c|c|c|c|c|c|c|c|}
\hline $\begin{array}{l}\text { Growth } \\
\text { stage }\end{array}$ & $\begin{array}{l}\text { Root morphology / } \\
\text { physiology parameters }\end{array}$ & $\begin{array}{l}\text { No. of } \\
\text { panicles }\end{array}$ & $\begin{array}{l}\text { Spikelets } \\
\text { per panicle }\end{array}$ & $\begin{array}{l}\text { Percentage of } \\
\text { filled grain }\end{array}$ & $\begin{array}{l}\text { Total spikelets } \\
\text { number }\end{array}$ & $\begin{array}{l}\text { 1000-grain } \\
\text { weight }\end{array}$ & Yield \\
\hline \multirow[t]{8}{*}{ MT } & Root dry weight & $-0.510^{*}$ & $0.595 * *$ & 0.257 & $0.447^{*}$ & $0.644 * *$ & $0.538 * *$ \\
\hline & Root length & $-0.473 *$ & $0.673 * *$ & 0.260 & $0.560 * *$ & $0.656^{* *}$ & $0.622 * *$ \\
\hline & Root-shoot ratio & -0.297 & $0.858 * *$ & $0.409^{*}$ & $0.853 * *$ & 0.372 & $0.849 * *$ \\
\hline & Root diameter & -0.278 & $0.891 * *$ & $0.470^{*}$ & $0.906^{* *}$ & $0.434 *$ & $0.920 * *$ \\
\hline & Specific root length & $0.417 *$ & $-0.442 *$ & -0.171 & -0.313 & $-0.529 * *$ & -0.386 \\
\hline & Root oxidation activity & -0.310 & $0.787 * *$ & 0.356 & $0.769 * *$ & $0.594 * *$ & $0.802 * *$ \\
\hline & Total absorbing surface area & -0.009 & $0.583 * *$ & 0.209 & $0.675 * *$ & 0.351 & $0.639 * *$ \\
\hline & Active absorbing surface area & -0.081 & $0.730 * *$ & $0.416^{*}$ & $0.810 * *$ & 0.371 & $0.818^{* *}$ \\
\hline \multirow[t]{8}{*}{ PI } & Root dry weight & $-0.477 *$ & $0.620 * *$ & 0.337 & $0.495 * *$ & $0.633^{* *}$ & $0.600 * *$ \\
\hline & Root length & $-0.457 *$ & $0.730 * *$ & 0.343 & $0.630 * *$ & $0.634 * *$ & $0.700 * *$ \\
\hline & Root-shoot ratio & 0.038 & $0.600 * *$ & 0.377 & $0.715^{* *}$ & 0.183 & $0.705 * *$ \\
\hline & Root diameter & -0.361 & $0.900 * *$ & $0.441^{*}$ & $0.872 * *$ & $0.483^{*}$ & $0.887 * *$ \\
\hline & Specific root length & 0.372 & $-0.462 *$ & -0.239 & -0.363 & $-0.525 * *$ & $-0.447^{*}$ \\
\hline & Root oxidation activity & -0.253 & $0.899 * *$ & $0.554 * *$ & $0.928 * *$ & $0.432 *$ & $0.960 * *$ \\
\hline & Total absorbing surface area & -0.241 & $0.843 * *$ & $0.412^{*}$ & $0.867 * *$ & $0.466^{*}$ & $0.868 * *$ \\
\hline & Active absorbing surface area & -0.247 & $0.864 * *$ & $0.460^{*}$ & $0.889 * *$ & $0.455^{*}$ & $0.903 * *$ \\
\hline \multirow[t]{8}{*}{ HD } & Root dry weight & -0.357 & $0.666^{* *}$ & 0.314 & $0.606^{* *}$ & $0.607 * *$ & $0.663 * *$ \\
\hline & Root length & -0.378 & $0.684 * *$ & 0.291 & $0.613 * *$ & $0.612 * *$ & $0.665^{* *}$ \\
\hline & Root-shoot ratio & -0.018 & $0.781 * *$ & $0.438^{*}$ & $0.906 * *$ & 0.109 & $0.839 * *$ \\
\hline & Root diameter & -0.249 & $0.841^{* *}$ & $0.515^{* *}$ & $0.856^{* *}$ & $0.455^{*}$ & $0.902 * *$ \\
\hline & Specific root length & 0.194 & $-0.540 * *$ & -0.299 & $-0.543 * *$ & $-0.505 * *$ & $-0.586^{* *}$ \\
\hline & Root oxidation activity & $-0.411^{*}$ & $0.883 * *$ & $0.488 * *$ & $0.832 * *$ & $0.496^{* *}$ & $0.873 * *$ \\
\hline & Total absorbing surface area & -0.089 & $0.790 * *$ & $0.661^{* *}$ & $0.870 * *$ & 0.313 & $0.940 * *$ \\
\hline & Active absorbing surface area & -0.117 & $0.815^{* *}$ & $0.627 * *$ & $0.887 * *$ & 0.319 & $0.942 * *$ \\
\hline \multirow[t]{8}{*}{ GF } & Root dry weight & -0.363 & $0.545 * *$ & 0.238 & $0.461 *$ & $0.621 * *$ & $0.531 * *$ \\
\hline & Root length & $-0.447 *$ & $0.639 * *$ & 0.255 & $0.529 * *$ & $0.627 * *$ & $0.591 * *$ \\
\hline & Root-shoot ratio & 0.263 & $0.437 *$ & 0.044 & $0.635^{* *}$ & 0.107 & $0.489 * *$ \\
\hline & Root diameter & -0.322 & $0.859 * *$ & $0.505 * *$ & $0.850 * *$ & $0.468^{*}$ & $0.896^{* *}$ \\
\hline & Specific root length & 0.096 & -0.364 & -0.224 & -0.384 & $-0.479 *$ & $-0.439 *$ \\
\hline & Root oxidation activity & $0.569 * *$ & -0.143 & 0.369 & 0.094 & -0.326 & 0.158 \\
\hline & Total absorbing surface area & -0.245 & $0.861 * *$ & $0.452^{*}$ & $0.879 * *$ & 0.404 & $0.885^{* *}$ \\
\hline & Active absorbing surface area & 0.076 & $0.629 * *$ & $0.486^{*}$ & $0.761 * *$ & 0.232 & $0.776^{* *}$ \\
\hline \multirow[t]{8}{*}{ MA } & Root dry weight & -0.362 & $0.509 * *$ & 0.207 & $0.421^{*}$ & $0.610 * *$ & $0.488^{* *}$ \\
\hline & Root length & $-0.470 *$ & $0.633 * *$ & 0.242 & $0.511^{* *}$ & $0.634 * *$ & $0.574 * *$ \\
\hline & Root-shoot ratio & 0.164 & 0.345 & -0.009 & $0.467 *$ & 0.292 & 0.376 \\
\hline & Root diameter & $-0.462 *$ & $0.826^{* *}$ & $0.432 *$ & $0.751 * *$ & $0.596 * *$ & $0.819 * *$ \\
\hline & Specific root length & 0.014 & -0.276 & -0.187 & -0.319 & $-0.409 *$ & -0.366 \\
\hline & Root oxidation activity & $0.672 * *$ & -0.331 & 0.156 & -0.088 & $-0.628 * *$ & -0.115 \\
\hline & Total absorbing surface area & 0.262 & -0.229 & 0.248 & -0.151 & -0.133 & -0.057 \\
\hline & Active absorbing surface area & 0.368 & $-0.536 * *$ & 0.035 & $-0.461 *$ & -0.276 & -0.384 \\
\hline
\end{tabular}




\subsection{Relationships of Root Traits With Grain Yield and Its Components}

Regression analysis showed that the root dry weight, root length, root-shoot ratio and root diameter of 24 mid-season rice cultivars from the mid-tillering to the grain filling stage were very significantly and positively correlated with grain yield ( $r=0.489$ to $0.920, \mathrm{P}<0.01$ ), whereas specific root length was negatively correlated with grain yield from panicle initiation to grain filling stage $(r=-0.586$ to $-0.439, \mathrm{P}<0.05)$. Root dry weight, root length and root-shoot ratio from the mid-tillering to the heading stage were also significantly and positively correlated with spikelets per panicle $(r=0.595$ to $0.858, \mathrm{P}<0.01)$. During the whole growth period, root diameter was significantly and positively correlated with spikelets per panicle, the percentage of filled grains, total spikelets number and grain weight $(r=0.432$ to $0.900, \mathrm{P}<0.05$ ) (Table 6).

From the mid-tillering to heading stage, root oxidation activity (ROA) was very significantly and positively correlated with grain yield $(r=0.802$ to $0.960, \mathrm{P}<0.01)$, and it was also significantly and positively correlated with spikelets per panicle, total spikelets number and grain weight $(r=0.432$ to $0.928, \mathrm{P}<0.05)$. From mid-tillering to grain filling stage, the total root absorbing surface area and active root absorbing surface area were all very significantly and positively correlated with grain yield $(r=0.639$ to $0.942, \mathrm{P}<0.01)$; from panicle initiation to grain filling stage, the total root absorbing surface area and active root absorbing surface area were significantly and positively correlated with spikelets per panicle, the total spikelets number and the percentage of filled grains $(r=$ 0.412 to $0.928, \mathrm{P}<0.05$ ) (Table 6 ).

\section{Discussion}

In this study, we observed that the root dry weight, root length and root diameter were increased with the GI. The analysis showed that root diameter was positively and significantly correlated with yield components, indicating that they are the most important morphological traits for yield increase during the GI in our study. In plants, the root hair is an important organ for the uptake of nutrients and water (Chao et al., 2002; Vance et al., 2003). However, sampling process will make massive losses of root hairs under field conditions (Ding et al., 2012), thus, root hairs were not determined in this experiment. It would be helpful to investigate root hairs in hydroponic conditions to reveal their functions during the GI. The present results showed that the root oxidation activity, total absorbing surface area and active absorbing surface area of roots were increased or significantly increased with the GI at early and middle growth stages, indicating that both ROA and root absorbing surface area play important roles in increasing grain yield. These results suggested that the morphological and physiological traits of roots have been improved during the GI.

The relationship of the morphological and physiological traits of rice roots with grain yield has been a hot topic in rice research (Yang et al., 2011). What morphological and physiological traits of roots should be for a high-yielding rice cultivar is still debating. Whether or not a larger root biomass and a stronger root activity would contribute to higher grain yield in rice remains an argument (Samejima et al., 2005; Yang et al., 2011). Earlier studies have showed that the rice root number, root weight, and root absorbing area were closely related with grain yield (Zhang et al., 2011, 2013; Yang et al., 2012). The root activity (ROA, dehydrogenase activity and root bleeding sap), specific root activity per spikelet (ROA $\times$ root weight $/$ total spikelets number), and spikelet-bleeding intensity (root bleeding/total spikelet number in given time) were significantly or very significantly and positively correlated with the grain filling rate, the percentage of filled grains, 1000-grain weight, and grain yield (Pan et al., 1996; Sun et al., 2002; Zhang et al., 2006). However, contradict results were also reported recently. For example, Passioura (1983) suggested that roots, as a plant part involved in acquisition of nutrients and water, also consume photosynthates from the aboveground parts for their establishment and growth. Furthermore, the energy consumption to produce per unit dry weight was as twice for producing roots as for producing shoots. Based on this understanding, someone proposed the opinion of "root redundant growth", that is, excessive root biomass or a too large root-shoot ratio will bring adverse effects to grain yield due to the excessive energy consumption (Cai et al., 2003; Liu et al., 2002; Wang et al., 2004). Our results herein demonstrated that the root dry weight, root length, root diameter, root oxidation activity, and total root absorbing surface area and active absorbing surface area of 24 mid-season rice cultivars were very significantly and positively correlated with grain yield, indicating no redundant root growth under this experiment condition. These results suggested that the improvement in root biomass and activity were crucial for rice yield increase during the GI. Earlier studies indicated that redundant root growth was obvious under the conditions of limited resources supply, such as water or nutrition, and removing some roots could benefit rice growth and increase rice yield (Cai et al., 2003; Liu et al., 2003; Wang et al., 2004). Whereas in this study, the nitrogen application $\left(240 \mathrm{~kg} \mathrm{ha}^{-1}\right)$ was abundant and the light intensity was high in the two study years (2008 and 2009), and therefore no redundant root growth was observed and an increase in root growth could increase grain yield. 
The present results showed that the increase in grain yield during the GI mainly resulted from the increase in total number of spikelets, leading to a larger sink size (total spikelets per unit area), especially in super hybrid rice. However, we also observed that the super rice cultivars bred in 2000s exhibited a low percentage of filled grains, which would limit their yield potential. Similar results were also reported by Yang and Zhang (2010), Yang (2011). The factors causing a low percentage of filled grains in rice cultivars with a large panicle size have been investigated, including source-sink relationships, matter translocation, and grain filling characteristics (Cao et al., 1981; Kobata et al., 1992; Yang et al., 1996; Gebbing et al., 1999; Samonte et al., 2001; Takai, et al., 2005; Peng et al., 2006). In this study, we observed that ROA, total root absorbing surface area and active absorbing surface area of super rice cultivars declined much faster than those of inbred cultivars during the grain filling period. These results suggest that the rapid senescence of roots might account for the low percentage of filled grains in super rice cultivars. We speculate that improvement in root activity during grain filling would increase the percentage of filled grains and consequently enhance yield potential in super rice.

It is noteworthy that rice yield in China has been increased significantly during the past several decades. Increase in fertilizer nutrient input, especially nitrogen fertilizer, has contributed significantly to the improvement of crop yields (Cassman et al., 2003). China's national average nitrogen rate for rice was $193 \mathrm{~kg} \mathrm{ha}^{-1}$ in 2006 (Peng et al., 2010). Nitrogen rates are ranged from 150 to $250 \mathrm{~kg} \mathrm{ha}^{-1}$, depending on rice planting areas (Wang et al., 2001; Peng et al., 2006). For the most high-yielding counties in Jiangsu province, China, the average nitrogen rate reached $300 \mathrm{~kg} \mathrm{ha}^{-1}$ (Liu et al., 2006). Under the nitrogen rate of $240 \mathrm{~kg} \mathrm{ha}^{-1}$ in this study, root traits and grain yield were all improved during the GI. The similar results were obtained when no nitrogen or low nitrogen rate $\left(120 \mathrm{~kg} \mathrm{ha}^{-1}\right)$ was applied (data not showed). Along with nitrogen rates, other cultivation techniques, such as tillage, planting density and transplanting methods, have also been changed in the past 60 years. These factors may also affect rice yield during the GI. Further study is needed to clarify the interactions between GI and nutrient management or agronomical improvement on root and shoot growth and grain yield of rice.

\section{Acknowledgments}

We are grateful for grants from the National Natural Science Foundation of China $(31271641 ; 31171481$; 31201155; 31371562), the National Basic Research Program (2012CB114306), the National Key Technology Support Program of China (2012BAD04B08), China National Public Welfare Industry (Agriculture) Plan (201203079; 201203031-02), the Priority Academic Program Development of Jiangsu Higher Education Institutions and Research Fund for the Doctoral Program of Higher Education in China (20123250120001).

\section{References}

Cai, K. Z., Luo, S. M., \& Duan, S. S. (2003). The response of the rice root system to nitrogen conditions under-root confinement. Acta Ecol. Sin., 23(6), 1109-1116.

Cao, X. Z., Zhu, Q. S., \& Gu, Z. F. (1981). Study on the percentage of filled grain in hybrid rice. Jiangsu Agric. Sci., 1, 1-7.

Cheng, S. H., Zhuang, J. Y., Fan, Y. Y., Du, J. H., \& Cao, L. Y. (2007). Progress in research and development on hybrid rice: A super-domesticate in China. Ann. Bot., 100, 959-966. http://dx.doi.org/10.1093/aob/mcm121

China National Rice Research Institute. (Eds.) (1988). Regionalization of rice cropping in China (pp. 1-47). Hangzhou: Zhejiang Science \& Technology Press.

Cho, H. T., \& Cosgrove, D. J. (2002). Regulation of root hair initiation and expansin gene expression in Arabidopsis. Plant Cell., 14, 3237-3253. http://dx.doi.org/10.1105/tpc.006437

Ding, W. N., Huang, W., Ning, Y. Q., \& Zhu, S. H. (2012). Genetic analysis and gene mapping of a novel short root hair mutant in rice. Acta Agron. Sin., 38(2), 240-244.

Dong, G. C., Wang, Y. L., Wang, J. G., Shan, Y. H., Ma, A. J., Yang, H. J., ... Cai, H. R. (2002). Study on the differences of root traits between various types of varieties in rice. Acta Agron. Sin., 28(6), 749-755.

Fitter, A. (2002). Characteristics and functions of root systems. Waise, L. Y., Eshel, A., Kafkafi, U., eds. Plant roots, the hidden half (pp. 15-32). New York: Marcel Dekker Inc.

Gebbing, T., \& Schnyder, H. (1999). Pre-anthesis reserve utilization for protein and carbohydrate synthesis in grains of wheat. Plant Physiol., 121, 871-878. http://dx.doi.org/10.1104/pp.121.3.871

Gu, J. F., Qiu, M., \& Yang, J. C. (2013). Enhanced tolerance to drought in transgenic rice plants overexpressing $\mathrm{C}_{4}$ photosynthesis enzymes. The Crop J., 1, 105-114. http://dx.doi.org/10.1016/j.cj.2013.10.002 
Gu, J. F., Yin, X. Y., Stomph, T. J., Wang, H. Q., \& Struik, P. C. (2012). Physiological basis of genetic variation in leaf photosynthesis among rice (Oryza sativa L.) introgression lines under drought and well-watered conditions. J. Exp. Bot., 63, 5137-5153. http://dx.doi.org/10.1093/jxb/ers170.

Gu, J. F., Yin, X. Y., Wang, H. Q., Struik, P. C., \& Stomph, T. J. (2014). Can exploiting natural genetic variation in leaf photosynthesis contribute to increasing rice productivity? A simulation analysis. Plant, Cell Environt., 37, 22-34. http://dx.doi.org/10.1111/pce.12173

Harada, J., Kang, S., \& Yamazaki, K. (1994). Root system development of japonica-indica hybrid rice cultivars. Jpn. J. Crop Sci., 63, 423-429.

Inukai, Y., Ashikari, M., \& Kitano, H. (2004). Function of the root system and molecular mechanism of crown root formation in rice. Plant Cell Physiol., 45(S), 17-19.

Kang, S. Y., Morita, S., \& Yamazaki, K. (1994). Root growth and distribution in some japonica-indica hybrid and japonica type rice cultivars under field conditions. Jpn. J. Crop Sci., 63, 118-124.

Kobata, T., Palta, J. A., \& Turner, N. C. (1992). Rate of development of post anthesis water deficits and grain-filling of spring wheat. Crop Sci., 32, 1238-1242.

Lawson, T., von Caemmerer, S., Baroli, I. (2011). Photosynthesis and Stomatal Behaviour. Prog. Bot., 72, 265-304. http://dx.doi.org/10.1007/978-3-642-13145-5_11

Ling, Q. H. (Eds.) (2000). Quality of crop population (pp. 42-107). Shanghai: Shanghai Scientific and Technical Publishers.

Liu, L. J., Xu, W., Sang, D. Z., Liu, C. L., Zhou, J. L., \& Yang, J. C. (2006). Site-specific nitrogen management increases fertilizer-nitrogen use efficiency in rice. Acta Agron. Sin., 32(7), 987-994.

Liu, T. J., Qi, C. H., \& Tang, J. J. (2002). Studies on relationship between the character parameters of root and yield formation in rice. Sci. Agric. Sin., 35(11), 1416-1419.

Liu, W. Z., \& Li, Y. Y. (2003). Effect of crop root-cutting on grain yield and water use efficiency: a review. Acta Bot. Boreali-Occident Sin., 23(8), 1320-1324.

Ota, Y. (1970). Diagnostic methods for the measurement of root activity in rice plant. Jpn Agric. Res. Quart., 5(3), $1-6$.

Pan, X. H., Wang, Y. R., \& Fu, J. R. (1996). Advance in the study on the growth-physiology in rice of root system (Oryza sativa). Chin. Bull. Bot., 13(2), 13-20.

Passioura, J. B. (1983). Roots and drought resistance. Agric. Water Manage., 7, 265-280.

Peng, S. B., Buresh, R. J., Huang, J. L., Yang, J. C., Zou, Y. B., Zhong, X. H., .. Zhang, F. S. (2006). Strategies for overcoming low agronomic nitrogen use efficiency in irrigated rice systems in China. Field Crops Res., 96, 37-47. http://dx.doi.org/10.1016/j.fcr.2005.05.004.

Peng, S. B., Buresh, R. J., Huang, J. L., Zhong, X. L., Zou, Y. B., Yang, J. C., ... Dobermann, A. (2010). Improving nitrogen fertilization in rice by site-specific N management. A review. Agron. Sustain. Dev., 30, 649-656. http://dx.doi.org/10.1051/agro/2010002.

Ramasamy, S., Ten Berge, H. F. M., \& Purushothaman, S. (1997). Yield formation in rice in response to drainage and nitrogen application. Field Crops Res., 51, 65-82. http://dx.doi.org/10.1016/S0378-4290(96)01039-8.

Samejima, H., Kondo, M., Ito, O., Nozoe, T., Shinano, T., \& Osaki, M. (2005). Characterization of root systems with respect to morphological traits and nitrogen-absorbing ability in the New Plant Type of tropical rice lines. J. Plant Nutr., 28, 835-850. http://dx.doi.org/10.1081/PLN-200055550.

Samonte, S., Wilson, L. T., McClung, A. M., \& Tarpley, L. (2001). Seasonal dynamics of non-structural carbohydrate partitioning in 15 diverse rice genotypes. Crop Sci., 41, 902-909.

Sun, J. W., Chen, W. F., Zang, C. M., Wang, Y. R., \& Wu, S. Q. (2002). Advances of research on rice root systems. J. Shenyang Agric. Univ., 33(6), 466-470.

Takai, T., Fukuta, Y., Shirawa, T., \& Horie, T. (2005). Time-related mapping of quantitative trait loci controlling grain-filling in rice. J. Exp. Bot., 56, 2107-2118. http://dx.doi.org/10.1093/jxb/eri209

Vance, C. P., Uhde-Stone, C., \& Allan, D. L. (2003). Phosphorus acquisition and use: critical adaptations by plants for securing a nonrenewable resource. New Phytol., 157, 423-447. http://dx.doi.org/10.1046/j.1469-8137. 2003.00695.x 
Wang, G. H., Dobermann, A., Witt, C., Sun, Q. Z., \& Fu, R. X. (2001). Performance of site-specific nutrient management for irrigated rice in southeast China. Agron. J., 93, 869-878. http://dx.doi.org/10.2134/agronj2001. 934869x

Wang, Q., Fan, X. L., Liu, F., Li, F. M., Klaus, D., \& Sattemacher, B. (2004). Effect of root cutting on rice yield by shifting normal paddy to upland cultivation. Chin. J. Rice Sci., 18(5), 437-442.

Wu, W. M., Song, X. F., Sun, Z. X., Yu, Y. H., \& Zou, G. Y. (2001). Comparison of root distribution between different type rice. Chin. J. Rice Sci., 15(4), 276-280.

Xiao, L. T., \& Wang, S. G. (2005). Experimental techniques of plant physiology (pp. 61-62). Beijing: China Agriculture Press.

Yang, J. C. (2010). Mechanism and regulation in the filling of inferior spikelets of rice. Acta Agron Sin., 36(12), 2011-2019.

Yang, J. C. (2011). Relationships of rice root morphology and physiology with the formation of grain yield and quality and the nutrient absorption and utilization. Sci. Agric. Sin., 44(1), 36-46.

Yang, J. C., \& Zhang, J. H. (2010). Grain filling problem in "super" rice. J. Exp. Bot., 61, 1-5. http://dx.doi.org/10.1093/jxb/erp348

Yang, J. C., Wang, P., Liu, L. J., Wang, Z. Q., \& Zhu, Q. S. (2006). Evolution characteristics of grain yield and plant type for mid-season indica rice cultivars. Acta Agron. Sin., 32(7), 949-955.

Yang, J. C., Wang, Z. Q., \& Zhu, Q. S. (1996). Effect of nitrogen nutrient on rice yield and its physiological mechanism under different status of soil moisture. Sci. Agric. Sin., 29(4), 58-66.

Yang, J. C., Zhang, H., \& Zhang, J. H. (2012). Root morphology and physiology in relation to the yield formation of rice. J. Integr. Agric., 11(6), 920-926. http://dx.doi.org/10.1016/S2095-3119(12)60082-3

Zhang, C. L., Jiang, W., Xiao, Y. Q., Wu, W. C., Chen, D. Z., \& Huang, Y. J. (2006). Status and prospects of research on rice root systems. Acta Agric. Jiangxi, 18(5), 23-27.

Zhang, H., Chen, T. T., Liu, L. J., Wang, Z. Q., Yand, J. C., \& Zhang, J. H. (2013). Performance in grain yield and physiological traits of rice in the Yangtze River Basin of China during the last $60 \mathrm{yr}$. J. Integr. Agric., 12(1), 57-66. http://dx.doi.org/10.1016/S2095-3119(13)60205-1.

Zhang, H., Huang, Z. H., Wang, J. C., Wang, Z. Q., \& Yang, J. C. (2011). Changes in morphological and physiological traits of roots and their relationships with grain yield during the evolution of mid-season Indica rice cultivars in Jiangsu Province. Acta Agron. Sin., 37(6), 1020-1030.

Zhang, H., Tan, G. L., Sun, X. L., Liu, L. J., \& Yang, J. C. (2009). Changes in grain quality during the evolution of mid-season indica rice cultivars in Jiangsu province. Acta Agron. Sin., 35(11), 2037-2044.

Zhang, Q. (2007). Strategies for developing green super rice. Proc. Natl. Acad. Sci. USA., 104, 16402-16409. http://dx.doi.org/10.1073/pnas.0708013104.

Zheng, J. S., \& Huang, Y. M. (2003). Thrust and practice of super high yielding rice production in China. Mol. Plant Breed., 1(5-6), 585-596.

Zhu, D. F., Lin, X. Q., \& Cao, W. X. (2000). Characteristics of root distribution of super high-yielding rice varieties. J. Nanjing Agric. Univ., 23(4), 5-8.

\section{Copyrights}

Copyright for this article is retained by the author(s), with first publication rights granted to the journal.

This is an open-access article distributed under the terms and conditions of the Creative Commons Attribution license (http://creativecommons.org/licenses/by/3.0/). 